\title{
Galactic evolution of nitrogen
}

\author{
G. Israelian ${ }^{1}$, A. Ecuvillon ${ }^{1}$, R. Rebolo ${ }^{1,2}$, R. García-López ${ }^{1,3}$, P. Bonifacio ${ }^{4}$, and P. Molaro ${ }^{4}$ \\ 1 Instituto de Astrofísica de Canarias, 38200 La Laguna, Tenerife, Spain \\ 2 Consejo Superior de Investigaciones Científcas, Spain \\ 3 Departamento de Astrofísica, Universidad de La Laguna, Av. Astrofísico Francisco Sánchez s/n, 38206 La Laguna, \\ Tenerife, Spain \\ ${ }^{4}$ Osservatorio Astronomico di Trieste, via G. B. Tiepolo 11, 34131 Trieste, Italy
}

Received 23 January 2004 / Accepted 23 March 2004

\begin{abstract}
We present detailed spectroscopic analysis of nitrogen abundances in 31 unevolved metal-poor stars analysed by spectral synthesis of the near-UV NH band at $3360 \AA$ observed at high resolution with various telescopes. We found that $[\mathrm{N} / \mathrm{Fe}]$ scales with that of iron in the metallicity range $-3.1<[\mathrm{Fe} / \mathrm{H}]<0$ with the slope $0.01 \pm 0.02$. Furthermore, we derive uniform and accurate $(\mathrm{N} / \mathrm{O})$ ratios using oxygen abundances from near- $\mathrm{UV}$ OH lines obtained in our previous studies. We find that a primary component of nitrogen is required to explain the observations. The NH lines are discovered in the VLT/UVES spectra of the very metal-poor subdwarfs G64-12 and LP815-43 indicating that these stars are N rich. The results are compared with theoretical models and observations of extragalactic H II regions and Damped Ly $\alpha$ systems. This is the first direct comparison of the $(\mathrm{N} / \mathrm{O})$ ratios in these objects with those in Galactic stars.
\end{abstract}

Key words. stars: abundances - galaxies: abundances

\section{Introduction}

Elemental abundance studies are important tracers of galaxy formation and evolution. The yields and origin of some important elements, such as nitrogen, are still a matter of serious debate. The stable isotope ${ }^{14} \mathrm{~N}$ is synthesized from ${ }^{12} \mathrm{C}$ and ${ }^{16} \mathrm{O}$ through the CNO cycles in a hydrogen-burning layer of a star. Knowledge of the origin of nitrogen is very important since, for example, this element can be used to derive the primordial helium abundance (Pagel \& Kazlauskas 1992). We call nitrogen "primary" when it is produced solely from the original hydrogen and helium in a star, either directly or through successive stages of nucleosynthesis. When the effective yield of nitrogen depends on the previous enrichment of $\mathrm{C}$ and $\mathrm{O}$, the nitrogen is considered as "secondary". Which stars produce secondary nitrogen is fairly well understood: non-zero metal-rich intermediate mass stars in the H-shell during the AGB phase (van den Hoek \& Groenewegen 1997). Freshly produced nitrogen may be brought to the surface by the third dredge-up and released in the interstellar medium by the stellar wind. Instead there are at least two possible sources of primary nitrogen:

1. intermediate mass which either undergo Hot Bottom Burning (Marigo 2001) or rotate (Meynet \& Maeder 2002);

2. metal-poor fast rotating massive stars where primary $\mathrm{N}$ is produced during the He-burning phase by rotational diffusion of ${ }^{12} \mathrm{C}$ into the He-burning layer (Meynet \& Maeder 2002).

Send offprint requests to: G. Israelian, e-mail: gil@ll.iac.es
The relative weight of secondary and primary components depends on the interplay between secondary enrichment caused by dredge up episodes and the primary contribution given by the CNO cycle during the envelope burning. It is well known that oxygen and perhaps also carbon are produced by massive stars (Maeder 1992). Several observations of the Galactic halo stars provided some support on the primary origin of $\mathrm{N}$ at low metallicities (Edmunds \& Pagel 1978; Pagel \& Edmunds 1981; Bessell \& Norris 1982; Tomkin \& Lambert 1984; Laird 1985; Carbon et al. 1987). However, it is commonly accepted that the main observational evidence of primary nitrogen at low metallicities is provided from abundance studies of $\mathrm{O}$ and $\mathrm{N}$ in H II regions of nearby metal-poor galaxies. Sargent \& Searle (1970) proposed that the most metal-poor galaxies are young systems experiencing their first star formation phase. At metallicities lower than $\log (\mathrm{O} / \mathrm{H}) \leq-4.0$ nitrogen is mostly primary with $\log (\mathrm{N} / \mathrm{O}) \simeq-1.5$ (see Henry et al. 2000, and references therein). This fact has led Thuan et al. (1995) to propose that $\mathrm{N}$ in these galaxies has been produced by massive stars as a primary element, and that therefore these stellar systems are young. However, recent spectrophotometric studies (e.g., Schulte-Ladbeck et al. 2001) of some metal-poor blue compact dwarf galaxies (BCGs) revealed old stellar populations. The cases of IZw 18 and SBS 0335-052 are still a matter of debate (i.e. Hunt et al. 2003). We also note that Henry et al. (2000) have proposed a different explanation for the low metallicity $(\mathrm{N} / \mathrm{O})$ plateau. These authors suggested that a very low star formation rate with massive stars producing $\mathrm{O}$ and intermediate mass stars producing primary $\mathrm{N}$ with a time-lag of $\sim 250 \mathrm{Myr}$ 
may produce a constant $(\mathrm{N} / \mathrm{O})$ as well. In fact, using the yields of N (van den Hoek \& Groenewegen 1997) and O (Maeder 1992) for a standard IMF, one obtains $\log (\mathrm{N} / \mathrm{O}) \sim-1.5$ at $\log (\mathrm{O} / \mathrm{H})<-4$ (Henry et al. 2000). Several authors have concluded that the contribution by massive stars to nitrogen is generally very low and the dominant primary $\mathrm{N}$ contributor is intermediate mass stars (Henry et al. 2000; Liang et al. 2001). However, others support the origin of primary nitrogen in massive stars (e.g., Thuan et al. 1995; Izotov et al. 2001) because of the low scatter of $(\mathrm{N} / \mathrm{O})$ ratios in galaxies observed at different stages of their evolution, which would imply no time delay between injection of nitrogen and oxygen. The scatter of (N/O) is large in high redshift low metallicity damped Ly $\alpha$ systems (DLAs) (Centurión et al. 1998; Lu et al. 1998; Pettini et al. 2002). However the sample can be split into two groups, each one with a relatively small dispersion (Centurión et al. $2003)$. The group with lower $(\mathrm{N} / \mathrm{O})$ ratio $(\log (\mathrm{N} / \mathrm{O})=-2.3)$ is about ten times lower than the bulk of DLAs and H II regions of BCGs.

The goal of this article is to perform a uniform and unbiased analysis of nitrogen and $(\mathrm{N} / \mathrm{O})$ ratios in a sample of Galactic metal-poor stars and compare them with theoretical models and many measurements available for extragalactic systems. We employed spectral synthesis of NH band in nearUV high-resolution spectra. Different spectral features can be used to measure the nitrogen abundance in solar-type stars. Weak high excitation $(\chi=10.34 \mathrm{eV})$ near-infrared $\mathrm{N}$ I lines at $7468.31,8216.34,8683.4,8703.25$ and $8718.83 \AA$ disappear at metallicities $[\mathrm{Fe} / \mathrm{H}]<-1$ and for the analysis of $\mathrm{N}$ in metal-poor stars one is left with the $\mathrm{CN}$ and $\mathrm{NH}$ molecular bands centered at 3883 and $3360 \AA$, respectively. The first studies of the NH band by Sneden (1973), Bessell \& Norris (1982) showed that one can use this spectral feature as an independent abundance indicator. Observations of the NH band at $3360 \AA$ have allowed many investigators to delineate the Galactic evolution of $\mathrm{N}$ down to $[\mathrm{Fe} / \mathrm{H}] \sim-3$ (Laird 1985; Carbon et al. 1987; Tomkin \& Lambert 1984; Israelian et al. 2000). Laird (1985) obtained constant $[\mathrm{N} / \mathrm{Fe}]=-0.67 \pm 0.14$ in the metallicity range $-2.5<[\mathrm{Fe} / \mathrm{H}]<0$ from the intermediate resolution spectra of 116 stars while Carbon et al. (1987) found $[\mathrm{N} / \mathrm{Fe}]= \pm 0.113 \pm 0.063$ from a similar-quality spectra of 76 subdwarfs. Unfortunately, NH lines are blended with several strong Ti and Sc lines and it is preferable to use high resolution spectra in order to avoid any systematics caused by the overabundance of Ti (an $\alpha$-element) and Sc in metal-poor stars. Tomkin \& Lambert (1984) used high resolution spectra of 14 metal-poor stars in the range $-2.3<[\mathrm{Fe} / \mathrm{H}]<-0.3$ and found $[\mathrm{N} / \mathrm{Fe}] \simeq-0.25$.

\section{Observations}

The near-UV spectra of our targets listed in Table 1 were obtained with the UES spectrograph, at the $4.2 \mathrm{~m}$ WHT (Observatorio del Roque de los Muchachos, La Palma), the UVES spectrograph, at the VLT/UT2 Kueyen Telescope (ESO, Chile), CASPEC at the $3.6 \mathrm{~m}$ ESO telescope (La Silla, Chile) and UCLES at the $3.9 \mathrm{~m}$ AAT (Australia). The spectra for these metal-poor stars have been collected and used to derive Be,
$\mathrm{O}, \mathrm{Cu}$ and $\mathrm{Zn}$ abundances in a series of papers (e.g., Molaro et al. 1987; Israelian et al. 1998, 2001; García López et al. 1995, 1998; Bihain et al. 2004). The high resolution spectra $(R \geq 50000$ in all the spectra except CASPEC which had $R \sim 35000$ ) near NH band $3360 \AA$ have $S / N$ ratios normally higher than 80 . The $S / N$ ratio was in the range $250-350$ in the VLT/UVES spectra of G64-12, G275-4 and LP815-43. The spectra were normalized using a 5 th order polynomial of the CONTINUUM task of IRAF. This normalization was good enough in all our targets with $[\mathrm{Fe} / \mathrm{H}]<-1$. However, the spectra of more metal-rich stars were normalized with regard to the solar spectrum of Kurucz et al. 1984 as described in Ecuvillon et al. (2004).

\section{Analysis}

\subsection{Rationale}

Our goal is to perform a uniform and unbiased analysis of the $(\mathrm{N} / \mathrm{O})$ ratio. Abundances of these elements can be determined from several molecular and atomic features. Both elements have high excitation atomic lines in the near IR and low excitation diatomic molecular bands in the near-UV. Many authors have compared abundances from $\mathrm{O}$ I against $\mathrm{OH}$ and $\mathrm{C}$ I against CH (Israelian et al. 1998, 2001; Boesgaard et al. 1999; Nissen et al. 2002; Tomkin et al. 1992) in metal-poor stars. While some authors found good agreement and others did not, it was realized that such comparisons are very sensitive to the stellar parameters. In fact, both $\mathrm{O}$ and $\mathrm{C}$ have abundance indicators that are less sensitive to the stellar parameters and almost independent on the non-LTE effects (i.e. [O I] 6300 and [C I] $8727 \AA$ ). A careful comparison of all three abundance indicators of $\mathrm{O}$ has been carried out for only few stars and positive results have been obtained (Israelian et al. 2001; Nissen et al. 2002). No detailed analysis has ever been carried out for Nitrogen because the high excitation lines of N I disappear at metallicities below $[\mathrm{Fe} / \mathrm{H}]<-1$. We note a good agreement found between the near-UV NH band and the high excitation N I $7468 \AA$ Aine in metal-rich stars (Ecuvillon et al. 2004). Even armed with different abundance indicators and high quality observations, we often face situations where little can be done to resolve apparent abundance conflicts (e.g., Takeda 2003; Fulbright \& Johnson 2003; Israelian et al. 2004). New generation 3D models of atmospheres were called in to save the situation (Asplund \& Garcia-Perez 2001; Asplund et al. 2004). These models suggest that diatomic molecules (e.g. OH, $\mathrm{CH}$ and $\mathrm{NH}$ ) in the near UV strongly overestimate elemental abundances derived using 1D models. The effect is strong in hot and very metal-poor subdwarfs. However, 3D models do not provide consistent abundances from the near-UV OH, O I 7771-5 ^ and [O I] $6300 \AA$ lines (Nissen et al. 2002). These models are not calibrated and no grid is available to check them against observations of many solar-type stars with different atmospheric parameters. The models have yet to demonstrate that they can explain various observational characteristics (such as Balmer line profiles, continuum energy distribution, consistent abundances from different abundance indicators) in many stars with different parameters. 
Table 1. Nitrogen and oxygen abundances from NH band synthesis and from literature, respectively, for metal-poor stars.

\begin{tabular}{|c|c|c|c|c|c|c|c|}
\hline Star & $\begin{array}{l}T_{\text {eff }} \\
(\mathrm{K})\end{array}$ & $\begin{array}{c}\log g \\
\left(\mathrm{~cm} \mathrm{~s}^{-2}\right)\end{array}$ & $\begin{array}{c}\xi_{t} \\
\left(\mathrm{~km} \mathrm{~s}^{-1}\right)\end{array}$ & {$[\mathrm{Fe} / \mathrm{H}]$} & {$[\mathrm{N} / \mathrm{H}]$} & {$[\mathrm{O} / \mathrm{H}]$} & {$[\mathrm{N} / \mathrm{O}]$} \\
\hline LP 815-43 & $6265 \pm 125$ & $4.54 \pm 0.30$ & 1.00 & -2.74 & $<-2.44$ & $-2.11 \pm 0.27^{2}$ & $<-0.33$ \\
\hline LHS 540 & $5993 \pm 71$ & $3.88 \pm 0.20$ & 1.00 & -1.48 & $-1.58 \pm 0.16$ & - & - \\
\hline $\mathrm{BD}+23^{\circ} 3130$ & $5154 \pm 58$ & $2.93 \pm 0.28$ & 1.25 & -2.66 & $-2.86 \pm 0.16$ & $-1.78 \pm 0.17^{2}$ & -1.08 \\
\hline $\mathrm{BD}+23^{\circ} 3912$ & $5734 \pm 67$ & $3.83 \pm 0.13$ & 1.00 & -1.50 & $-1.80 \pm 0.15$ & $-0.94 \pm 0.17^{3}$ & -0.86 \\
\hline $\mathrm{BD}+26^{\circ} 3578$ & $6263 \pm 76$ & $3.93 \pm 0.21$ & 1.00 & -2.36 & $<-1.36$ & $-1.42 \pm 0.11^{3}$ & $<-0.06$ \\
\hline $\mathrm{BD}+37^{\circ} 1458$ & $5326 \pm 54$ & $3.30 \pm 0.23$ & 1.25 & -2.17 & $-1.97 \pm 0.15$ & $-1.36 \pm 0.21^{1}$ & -0.61 \\
\hline G64-12 & $6318 \pm 150$ & $4.20 \pm 0.30$ & 1.00 & -3.05 & $-1.90 \pm 0.25$ & $-1.88 \pm 0.31^{2}$ & -0.02 \\
\hline G275-4 & $6212 \pm 150$ & $4.13 \pm 0.30$ & 1.00 & -2.99 & $<-2.29$ & $-2.08 \pm 0.32^{2}$ & $<-0.21$ \\
\hline HD 6582 & $5296 \pm 67$ & $4.46 \pm 0.05$ & 1.00 & -0.86 & $-1.05 \pm 0.14$ & $-0.53 \pm 0.16^{1}$ & -0.52 \\
\hline HD 19445 & $6095 \pm 69$ & $4.45 \pm 0.05$ & 1.00 & -2.04 & $-1.69 \pm 0.12$ & $-0.82 \pm 0.17^{1}$ & -0.87 \\
\hline HD 64090 & $5417 \pm 65$ & $4.55 \pm 0.06$ & 1.00 & -1.74 & $-1.75 \pm 0.14$ & $-1.00 \pm 0.15^{1}$ & -0.75 \\
\hline HD 76932 & $5859 \pm 72$ & $4.13 \pm 0.04$ & 1.00 & -0.90 & $-1.12 \pm 0.15$ & $-0.56 \pm 0.18^{1}$ & -0.56 \\
\hline HD 84937 & $6277 \pm 75$ & $4.03 \pm 0.09$ & 1.00 & -2.06 & $<-2.06$ & $-1.29 \pm 0.17^{1}$ & $<-0.77$ \\
\hline HD 87140 & $5086 \pm 43$ & $2.96 \pm 0.31$ & 1.25 & -1.83 & $-1.83 \pm 0.15$ & - & - \\
\hline HD 94028 & $6058 \pm 72$ & $4.27 \pm 0.06$ & 1.00 & -1.49 & $-1.34 \pm 0.12$ & $-0.78 \pm 0.16^{1}$ & -0.56 \\
\hline HD 103095 & $5047 \pm 68$ & $4.61 \pm 0.05$ & 1.00 & -1.43 & $-1.83 \pm 0.14$ & $-1.06 \pm 0.19^{3}$ & -0.77 \\
\hline HD 128279 & $5130 \pm 66$ & $2.85 \pm 0.22$ & 1.25 & -2.11 & $-2.41 \pm 0.16$ & - & - \\
\hline HD 134169 & $5877 \pm 72$ & $3.96 \pm 0.07$ & 1.00 & -0.81 & $-0.81 \pm 0.15$ & $-0.52 \pm 0.17^{1}$ & -0.29 \\
\hline HD 140283 & $5723 \pm 67$ & $3.68 \pm 0.06$ & 1.00 & -2.47 & $-2.42 \pm 0.11$ & $-1.58 \pm 0.15^{1}$ & -0.84 \\
\hline HD 157214 & $5715 \pm 43$ & $4.21 \pm 0.03$ & 1.00 & -0.35 & $-0.45 \pm 0.12$ & $-0.24 \pm 0.11^{1}$ & -0.21 \\
\hline HD 165908 & $5905 \pm 42$ & $4.11 \pm 0.03$ & 1.00 & -0.67 & $-0.77 \pm 0.12$ & $-0.63 \pm 0.10^{1}$ & -0.14 \\
\hline HD 166913 & $6181 \pm 74$ & $4.18 \pm 0.07$ & 1.00 & -1.68 & $-1.38 \pm 0.12$ & $-0.69 \pm 0.18^{1}$ & -0.69 \\
\hline HD 170153 & $5954 \pm 42$ & $4.13 \pm 0.03$ & 1.00 & -0.65 & $-0.86 \pm 0.12$ & $-0.74 \pm 0.12^{1}$ & -0.12 \\
\hline HD 188510 & $5559 \pm 65$ & $4.54 \pm 0.06$ & 1.00 & -1.57 & $-1.57 \pm 0.14$ & $-1.01 \pm 0.17^{1}$ & -0.56 \\
\hline HD 189558 & $5681 \pm 70$ & $3.81 \pm 0.08$ & 1.00 & -1.12 & $-1.32 \pm 0.15$ & $-0.74 \pm 0.17^{1}$ & -0.58 \\
\hline HD 194598 & $6029 \pm 77$ & $4.28 \pm 0.07$ & 1.00 & -1.20 & $-1.10 \pm 0.15$ & $-0.68 \pm 0.19^{1}$ & -0.42 \\
\hline HD 201889 & $5618 \pm 67$ & $4.04 \pm 0.08$ & 1.00 & -0.95 & $-1.05 \pm 0.14$ & $-0.80 \pm 0.20^{1}$ & -0.25 \\
\hline HD 201891 & $5916 \pm 77$ & $4.26 \pm 0.05$ & 1.00 & -1.05 & $-0.95 \pm 0.15$ & $-0.60 \pm 0.18^{1}$ & -0.35 \\
\hline HD 218502 & $6182 \pm 77$ & $4.08 \pm 0.08$ & 1.00 & -1.76 & $<-2.06$ & - & - \\
\hline HD 211998 & $5282 \pm 180$ & $3.28 \pm 0.28$ & 1.25 & -1.48 & $-1.98 \pm 0.30$ & $-1.27 \pm 0.38^{1}$ & -0.71 \\
\hline HD 225239 & $5528 \pm 44$ & $3.74 \pm 0.15$ & 1.00 & -0.50 & $-0.65 \pm 0.09$ & $-0.71 \pm 0.12^{1}$ & 0.06 \\
\hline
\end{tabular}

${ }^{1}$ From Israelian et al. (1998).

${ }^{2}$ From Israelian et al. (2001).

${ }^{3}$ From Boesgaard et al. (1999).

To ensure a homogeneous study of the $(\mathrm{N} / \mathrm{O})$ ratio from the $\mathrm{NH}$ and $\mathrm{OH}$ features in the near-UV, we used the same model atmospheres and tools as in our previous studies (e.g., Israelian et al. 1998, 2001). The oxygen abundances were compiled from the papers of Israelian et al. $(1998,2001)$ and Boesgaard et al. (1999). Few stars have been added to the lists of Israelian et al. $(1998,2001)$ while the stellar parameters and $[\mathrm{Fe} / \mathrm{H}]$ (Table 1) were taken from Bihain et al. (2004). The effective temperatures were computed by Bihain et al. (2004) using the IRFM calibration versus $V-K$ colors, the gravities from Hipparcos parallaxes and the metallicities from the literature. The parameters of G64-12, G275-4 and LP815-43 were taken from Israelian et al. (2001). Uncertainties in the atmospheric parameters are of the order of $80 \mathrm{~K}$ in $T_{\text {eff }}, 0.1$ in $\log g$ and 0.15 in the metallicity. The effect of $T_{\text {eff }}, \log g$ and $[\mathrm{Fe} / \mathrm{H}]$ on the $\mathrm{NH}$ abundance was considered by Ecuvillon et al. (2004). We found that the effect is not different in metal-poor stars and assumed the same sensitivity values as in Ecuvillon et al. (2004). The sensitivity of $\mathrm{NH}$ abundance was applied to the propagation of the error of each parameter error on abundances. A continuum uncertainty effect of the order of 0.1 dex was considered in all targets except for three stars observed with VLT/UVES where the error was less than 0.05 dex. All errors were added in quadrature to obtain final uncertainties for nitrogen abundances listed in Table 1.

\subsection{Synthesis of $\mathrm{NH}$ band}

Nitrogen abundances were determined by fitting an LTE synthetic spectrum to data in the region $\lambda \lambda 3345-3375 \AA$. A detailed line list from Ecuvillon et al. (2004) was employed. These authors have slightly modified the line list of Yakovina \& Pavlenko (1998) without applying any change to the continuum level when fitting the high resolution Kurucz Solar Atlas (Kurucz et al. 1984) with a solar model having $T_{\text {eff }}=5777 \mathrm{~K}$, $\log g=4.44$ and $\xi_{t}=1.0 \mathrm{~km} \mathrm{~s}^{-1}$. To measure the $\mathrm{N}$ abundance in stars with $-1<[\mathrm{Fe} / \mathrm{H}]<0$ we used the program FITTING ${ }^{1}$.

${ }^{1}$ FITTING is developed by Jonay I. González-Hernández (Instituto de Astrofísica de Canarias). 

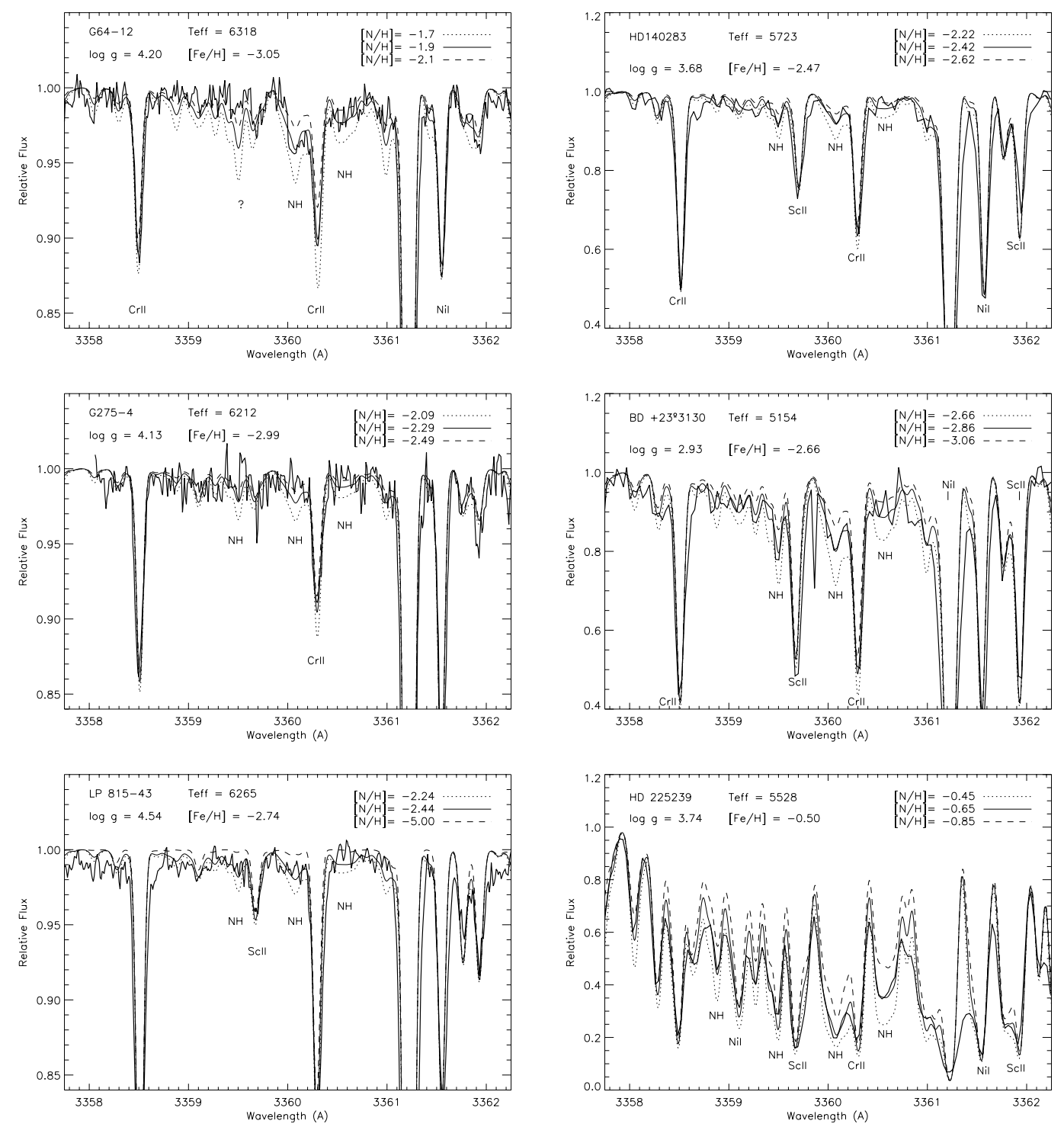

Fig. 1. Observations and synthesis of the NH band in the high-resolution near-UV spectra of several metal-poor stars.

This program creates a grid of synthetic spectra computed with MOOG (Sneden 1974) for several free parameters (e.g., abundances, atmospheric parameters, etc.) and compares them with the observed spectrum. The best fit is determined by applying a $\chi^{2}$ minimization method to the spectral regions considered most significant (Ecuvillon et al. 2004). Instrumental broadening was computed using a Gaussian function corresponding to the resolution of the instrument, while the value of $v \sin i$ was adjusted by eye and was always less than $3 \mathrm{~km} \mathrm{~s}^{-1}$ since all our targets are evolved slow rotators. Our tests with the $\chi^{2}$ technique show that the nitrogen abundance (as well as the abundances of all other elements) is not affected by $v \sin i$. The fits were performed by scaling the abundances of all elements to the $[\mathrm{Fe} / \mathrm{H}]$ value and then modifying them typically within \pm 0.4 dex until the best solution was found. It was much easier to derive the nitrogen abundance in stars with $[\mathrm{Fe} / \mathrm{H}]<-2$, where most of the blends disappear and the position of continuum can be determined with better precision. The goodness of the spectral synthesis in these stars was estimated by eye.
In Fig. 1 we show the results of the fits for six of our program stars which span the full range of metallicities covered by our sample. In each panel the observed spectrum of a star is compared with the best fitting synthetic spectrum and with two other synthetic spectra with different $\mathrm{N}$ abundance which bracket the best fitting spectrum. Such plots allow to estimate the errors associated to the $\mathrm{N}$ abundance measurement. It is noticeable in this plot that the most metal-poor star in our sample, G64-12, has a clearly measurable NH band. As will be stated in a well defined sense in Sect. 4.4, this star appears to be N-rich $([\mathrm{N} / \mathrm{Fe}]=+1.15)$. Stars LP815-43 and G275-4 (first and second left panel from the bottom) form an interesting pair: their atmospheric parameters are very similar, yet the NH band is measurable in the former, but not in the latter. Neither of these stars is known to be in a binary system. LP815-43 ([N/Fe] = +0.63), like G64-12, belongs to the group of $\mathrm{N}$-rich stars. From the upper right panel one can see that we can confirm the detection of NH band in HD 140283 (Bessell \& Norris 1982; Tomkin \& Lambert 1984). From the spectrum of 

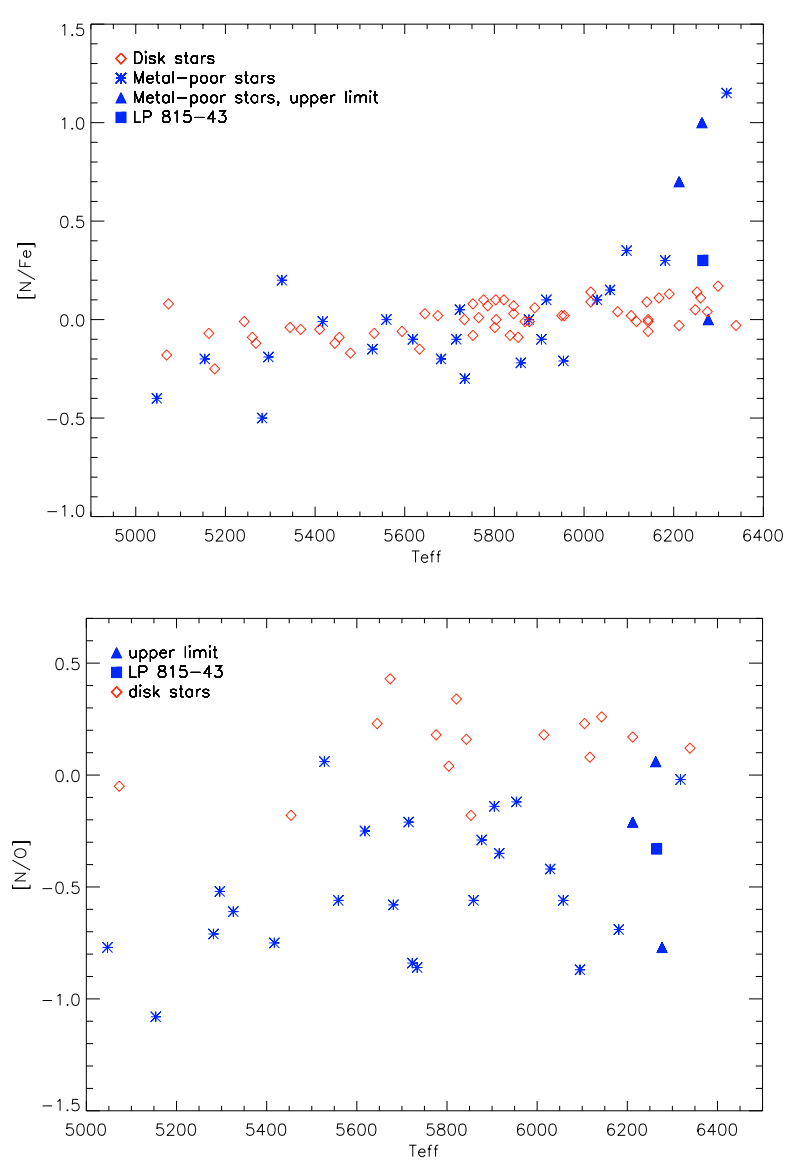

Fig. 2. $[\mathrm{N} / \mathrm{Fe}]$ and $[\mathrm{N} / \mathrm{O}]$ ratios versus $T_{\text {eff }}$ and $\log g$.

HD 225239 (lower right panel) it appears clear how the analysis of the $\mathrm{NH}$ is more difficult at higher metallcities due to the strong blending with metallic lines and saturation of the $\mathrm{NH}$ lines.

The solar abundance of nitrogen was assumed log $\epsilon(\mathrm{N})=8.05$ (on the customary scale in which $\log \epsilon(\mathrm{H})=12$ ) from Anders \& Grevesse (1989). There is, however, some debate over the oxygen abundance in the Sun (Allende Prieto et al. 2001; Nissen et al. 2002; Asplund et al. 2004). The latest value, $\log \epsilon(\mathrm{O})=8.66$, from $3 \mathrm{D}$ models is about $0.3 \mathrm{dex}$ smaller than the original value from Anders \& Grevesse (1989). The solar oxygen abundance used for the differential analysis of $\mathrm{OH}$ lines by Israelian et al. (1998) was $\log \epsilon(\mathrm{O})=8.93$ from Anders \& Grevesse (1989). In order to be consistent with the solar $g f$ values used by Israelian et al. (1998) and with 1D model analysis in general, we have decided to use the same value in this article. This assumption will not introduce any errors since one can always switch to the scale where $\log \epsilon(\mathrm{O})=8.66$ or any other value discussed in the literature and accordingly modify solar $g f$ values of the $\mathrm{OH}$ lines from Israelian et al. (1998). The oxygen abundances in our targets come from the near-UV OH lines analysed by Israelian et al. $(1998,2001)$ and Boesgaard et al. (1999). The oxygen abundances listed in these articles were updated for the parameters listed in the Table 1 , using the sensitivity of $\mathrm{OH}$ to $T_{\text {eff }}, \log g$ and $[\mathrm{Fe} / \mathrm{H}]$ given in Israelian et al. (1998).

We analysed near-UV high-resolution spectra of 31 metalpoor stars with the goal of delineating Galactic trends
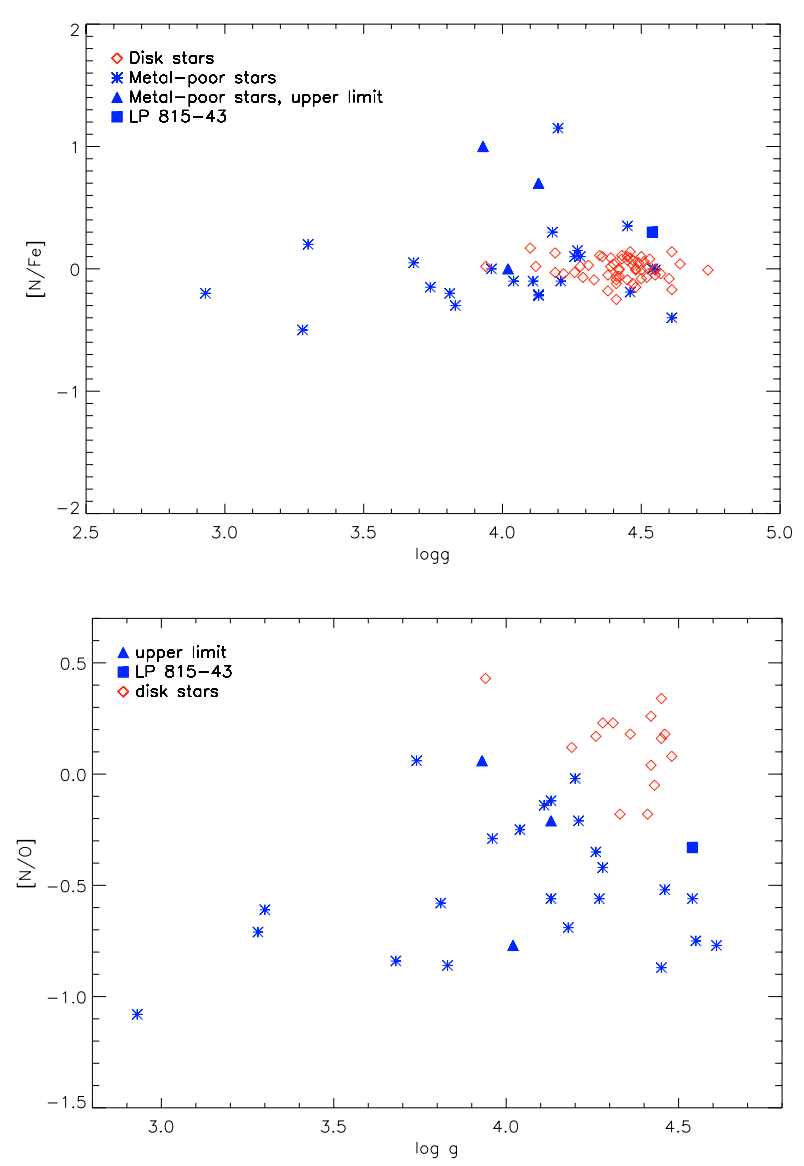

of $[\mathrm{N} / \mathrm{Fe}],[\mathrm{N} / \mathrm{H}]$ and $[\mathrm{N} / \mathrm{O}]$. All our targets are unevolved metal-poor stars with $[\mathrm{Fe} / \mathrm{H}]$ values between -0.3 and -3.1 and $T_{\text {eff }}$ between 5000 and $6300 \mathrm{~K}$. The results of our analysis are listed in Table 1. We have investigated the presence of the possible systematic dependence of $[\mathrm{N} / \mathrm{Fe}]$ and $[\mathrm{N} / \mathrm{O}]$ on $T_{\text {eff }}$ and $\log g$ in Fig. 2. The absence of any trend in these figures suggests that the uncertainties in the derived oxygen abundances are only related to the errors listed in Table 1. On the whole, the $[\mathrm{N} / \mathrm{Fe}]$ vs. $[\mathrm{Fe} / \mathrm{H}]$ trend appears to be almost flat with a slope of $0.01 \pm 0.02$ (without considering G64-12, LP815-43 and the upper limits). In Fig. 3 we plot the $[\mathrm{N} / \mathrm{O}]$ ratios in our progran stars as a function of $[\mathrm{O} / \mathrm{H}],[\mathrm{Fe} / \mathrm{H}]$ and $[\mathrm{N} / \mathrm{H}]$. Like in Fig. 2 the diamond shapes are the $\mathrm{N}$ measurements from Ecuvillon et al. (2004). From all the three plots it is clear that the two stars G 64-12 and LP 815-43 have N/O ratios higher than other stars of similar metallicity, which justify their designation as N-rich. What is also apparent is that there is an increase in the N/O ratio with metallicity. To highlight this increase we plotted, as a dashed line, in each panel a linear regression, the slope of which is given at the bottom of each plot. In the two upper panels we also plotted, as a reference, a horizontal dotted line corresponds to $[\mathrm{N} / \mathrm{O}]=-0.62$, which is the level of the plateau in N/O observed in Blue Compact Galaxies (Izotov et al. 2000). Figure 4 shows that the $[\mathrm{N} / \mathrm{Fe}]$ ratio is constant whichever the metallicity, and that $[\mathrm{N} / \mathrm{H}]$ increases linearly with $[\mathrm{Fe} / \mathrm{H}]$. The inference from these plots is that nitrogen and iron vary in lockstep, in substantial agreement with the main conclusion of Carbon et al. (1987). 

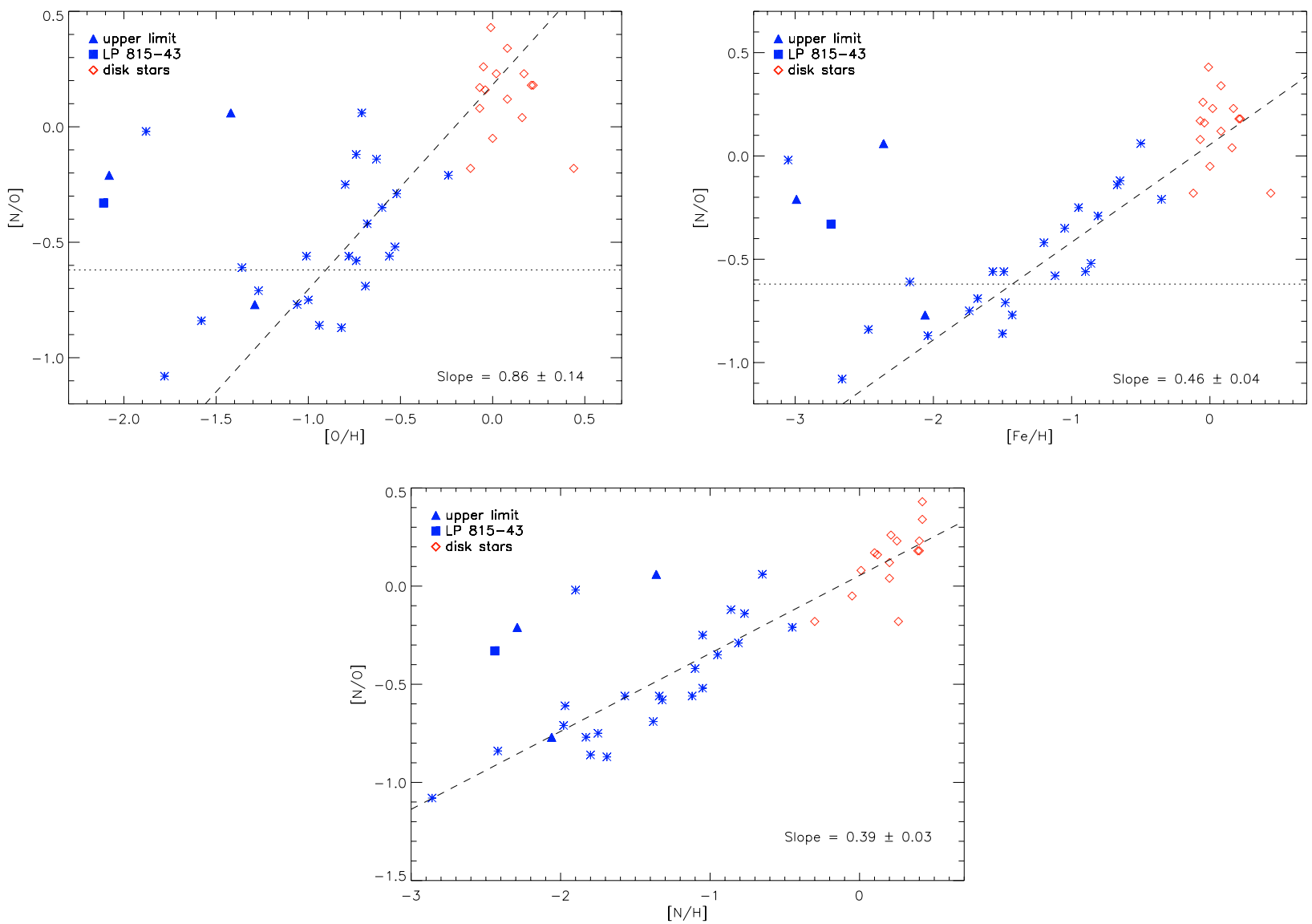

Fig. 3. The $[\mathrm{N} / \mathrm{O}]$ ratio as a function of $[\mathrm{O} / \mathrm{H}],[\mathrm{Fe} / \mathrm{H}]$ and $[\mathrm{N} / \mathrm{H}]$ for the programme stars and for the metal-rich disc stars of Ecuvillon et al. (2004). The dotted line represents $\log (\mathrm{N} / \mathrm{O}) \simeq-1.5$ from Henry et al. (2000) while the dashed line is a least-square fit to the data without taking into account $\mathrm{N}$-rich stars (see the text). A preliminary detection of $\mathrm{N}$ is reported in LP815-43.

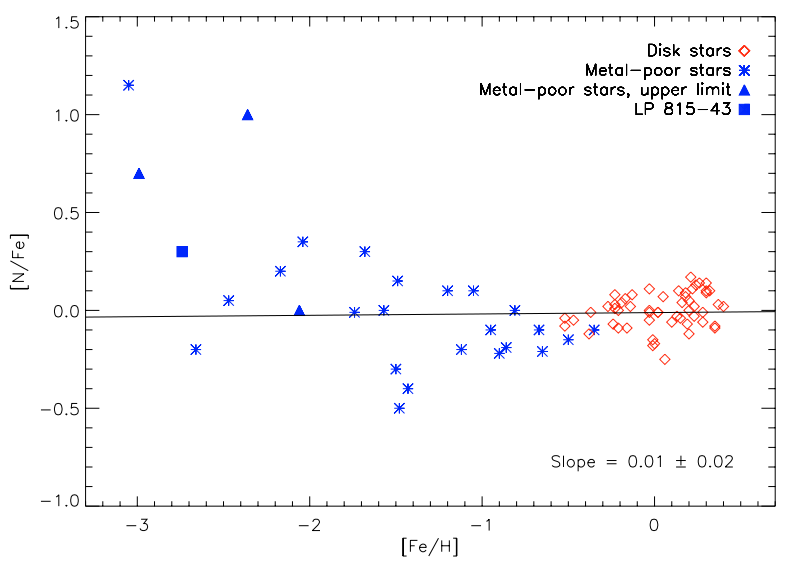

Fig. 4. $[\mathrm{N} / \mathrm{Fe}]$ and $[\mathrm{N} / \mathrm{H}]$ ratios against $[\mathrm{Fe} / \mathrm{H}]$.

In order to extend our plots to the regime of metal-rich stars we used NH abundance from Ecuvillon et al. (2004) and oxygen abundances from the literature (Santos et al. 2000; Takeda et al. 2001; Gonzalez et al. 2001; Sadakane et al. 2002). Oxygen abundances in these studies were obtained either from the triplet at 7771-5 $\AA$ or from the forbidden line at $6300 \AA$. We do not think this will introduce large errors in the final (N/O) ratios since different abundance indicators provide quite consistent abundances within \pm 0.2 dex in solar metallicity dwarfs. This is true for nitrogen (Ecuvillon et al. 2004) and oxygen

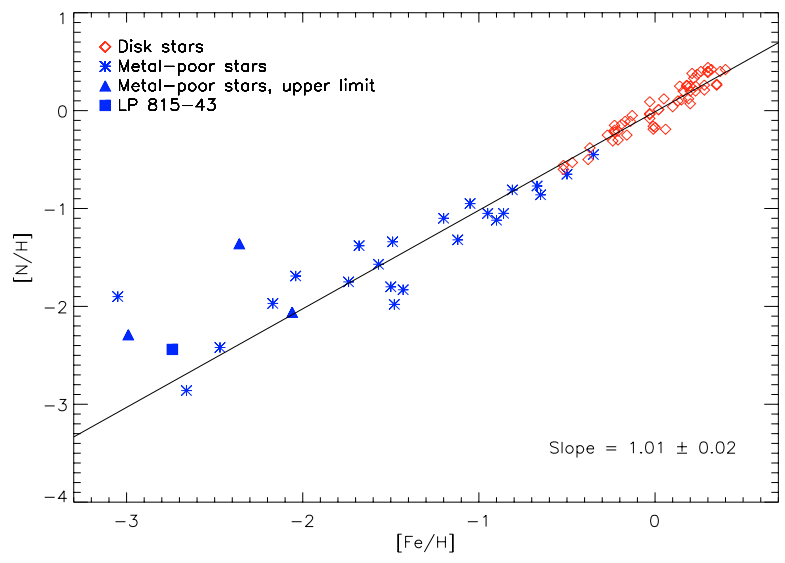

(Takeda 2003; Nissen et al. 2002). The compiled data are provided in Table 2.

We stress that possible abundance differences from $\mathrm{N}$ I and $\mathrm{NH}$ lines produced by uncertainties in the near-UV continuum, 3D and other effects are not critical for the present analysis. Such differences cancel out when forming abundance ratios from lines formed in the same atmospheric layer. Similar arguments led Tomkin \& Lambert (1984) to derive (N/C) ratio from the $\lambda 3360 \mathrm{NH}$ and $\lambda 4300 \mathrm{CH}$ bands. Since the $\mathrm{CH}, \mathrm{NH}$ and $\mathrm{OH}$ hydrids have similar dissocitation energies and line 
Table 2. Nitrogen and oxygen abundances from NH band synthesis (Ecuvillon et al. 2004) and from literature (last column), respectively, for metal-rich stars.

\begin{tabular}{lccccccc}
\hline \hline Star & $\begin{array}{c}T_{\text {eff }} \\
(\mathrm{K})\end{array}$ & $\begin{array}{c}\log g \\
\left(\mathrm{~cm} \mathrm{~s}^{-2}\right)\end{array}$ & $\begin{array}{c}\xi_{t} \\
\left(\mathrm{~km} \mathrm{~s}^{-1}\right)\end{array}$ & {$[\mathrm{Fe} / \mathrm{H}]$} & {$[\mathrm{N} / \mathrm{H}]$} & {$[\mathrm{O} / \mathrm{H}]$} & Ref. \\
\hline HD 52265 & 6105 & 4.28 & 1.36 & 0.23 & 0.25 & 0.02 & 1 \\
HD 75289 & 6143 & 4.42 & 1.53 & 0.28 & 0.21 & -0.05 & 1 \\
HD 82943 & 6015 & 4.46 & 1.13 & 0.30 & 0.39 & 0.21 & 1 \\
HD 83443 & 5454 & 4.33 & 1.08 & 0.35 & 0.26 & 0.44 & 1 \\
HD 9826 & 6212 & 4.26 & 1.69 & 0.13 & 0.10 & -0.07 & 2 \\
HD 22049 & 5073 & 4.43 & 1.05 & -0.13 & -0.05 & 0.00 & 2 \\
HD 38529 & 5674 & 3.94 & 1.38 & 0.40 & 0.42 & -0.01 & 2 \\
HD 92788 & 5821 & 4.45 & 1.16 & 0.32 & 0.42 & 0.08 & 3 \\
HD 120136 & 6339 & 4.19 & 1.70 & 0.23 & 0.20 & 0.08 & 2 \\
HD 134987 & 5776 & 4.36 & 1.09 & 0.30 & 0.40 & 0.22 & 4 \\
HD 143761 & 5853 & 4.41 & 1.35 & -0.21 & -0.30 & -0.12 & 2 \\
HD 209458 & 6117 & 4.48 & 1.40 & 0.02 & 0.01 & -0.07 & 3 \\
HD 217014 & 5804 & 4.42 & 1.20 & 0.20 & 0.20 & 0.16 & 4 \\
HD 217107 & 5645 & 4.31 & 1.06 & 0.37 & 0.40 & 0.17 & 2 \\
HD 222582 & 5843 & 4.45 & 1.03 & 0.05 & 0.12 & -0.04 & 4 \\
\hline
\end{tabular}

\footnotetext{
${ }^{1}$ From Santos et al. (2000).

${ }^{2}$ From Takeda et al. (2001).

${ }^{3}$ From Sadakane et al. (2002).

${ }^{3}$ From Gonzalez et al. (2001).
}

formation mechanisms, the derived CNO abundance ratios are insensitive to atmospheric parameters and structure. Thus, we measure the $(\mathrm{N} / \mathrm{O})$ ratio using the lines of $\mathrm{NH}$ and $\mathrm{OH}$ in the near UV.

\section{Discussion and conclusions}

\subsection{Comparison with simple models}

From our data we can make a few statements that are quite robust in the sense that errors in the data cannot invalidate them:

1. The N/O ratio has an increasing trend together with both oxygen and nitrogen abundance (Fig. 3).

2. The nitrogen abundance varies in lockstep with that of iron (Fig. 4).

3. There are a few stars that have a N/O ratio higher than expected from this trend and their oxygen or nitrogen abundance. We shall refer to these stars as N-rich stars.

Let us now try to see if from these facts we may reach some conclusions with regard to the nucleosynthesis of nitrogen. Keeping in mind what has been said above concerning $\mathrm{N}$ nucleosynthesis, let us compare our data with some simple models. In Fig. 5 we plot our data and the simple closed box models of Meynet \& Maeder (2002) and the analytical models of Henry et al. (2000). The lower envelope of the data seems to lie in the region that could be adequately described by the models without any effect of stellar rotation. The remaining data, however, seem to require models with rotation to be explained. At low metallicities the Galactic stars clearly deviate from the $(\mathrm{N} / \mathrm{O})$ plateau which characterizes extragalactic $\mathrm{H}$ II regions (i.e. BCGs) and is described by the middle curve of the three analytical models of Henry et al. (2000). The data however could be described by the Henry et al. (2000) with a rather high star formation efficiency (the lowest of the three curves). Since both the Henry et al. (2000) and Meynet \& Maeder (2002) models include nitrogen of both primary and secondary origin, we believe it is safe to conclude that some primary $\mathrm{N}$ production is necessary to explain the data. By looking at the models of Meynet \& Maeder (2002) alone it might be tempting to conclude that inclusion of stellar rotation is necessary. However this is not necessarily so; in fact in the models of Henry et al. (2000) the yields for massive stars are taken from Maeder (1992), which do not include rotation. It is beyond the scope of this paper to make a detailed comparison with chemical evolution models; however, we believe that it is quite likely that any model with both primary and secondary nitrogen production would be able of reproducing the low metallicity data simply by tuning star formation and infall. In Fig. 5 there is a group of stars around $\log (\mathrm{O} / \mathrm{H})+12=8.2, \log (\mathrm{N} / \mathrm{O})=-1.1$ which seems to stand above all the models, and, although the errors are such that it is not inconsistent with the models.

The comparison with the models also suggests that there might be some intrinsic dispersion in N/O ratios at any given $\mathrm{O}$ abundance. The existence of such a dispersion would support the fact that AGB stars are the main contributor of primary nitrogen at low metallicities (Pagel \& Edmunds 1981; Pettini et al. 2002). This would also would support the role of rotation in $\mathrm{N}$ production; in fact, different $\mathrm{N}$ yields are predicted for different starting rotational velocities. Therefore, scatter in $\mathrm{N} / \mathrm{O}$ ratios would naturally arise from a distribution of initial rotational velocities. Differences in star formation history will introduce a scatter as well. The errors on the data, however, are too large to decide whether such an intrinsic dispersion exists. A mild hint in this direction comes from the inspection of the behaviour of the $\mathrm{N} / \mathrm{O}$ ratio as a function of $\mathrm{O}$ and $\mathrm{N}$ 


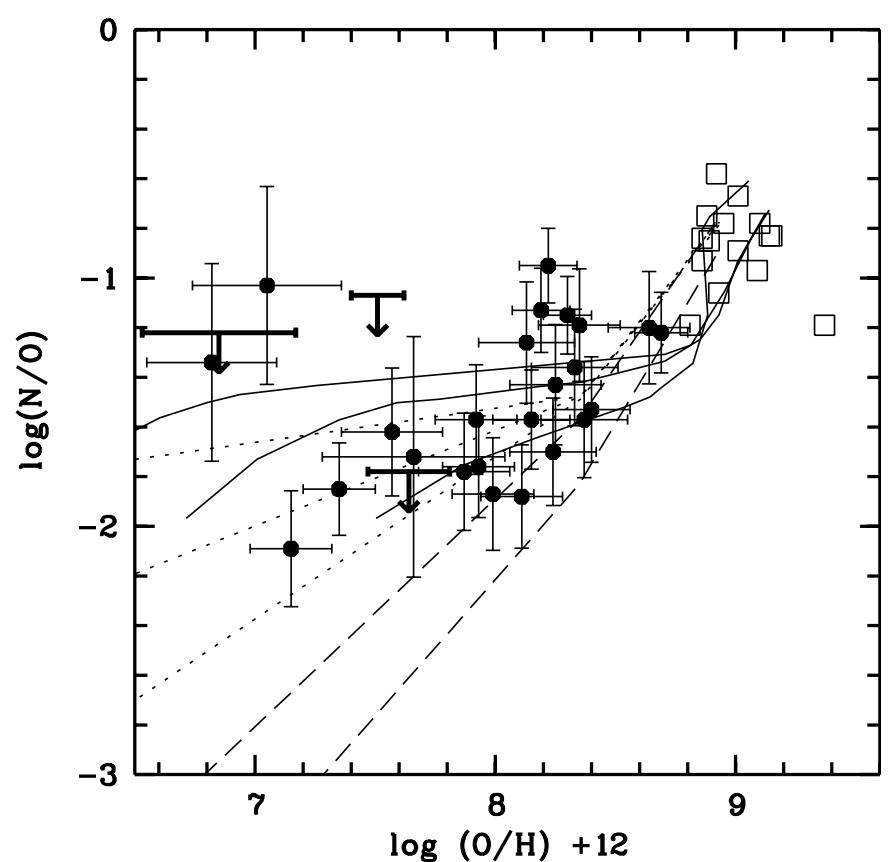

Fig. 5. Comparison of N/O ratios for programme stars (filled circles) with the simple models of Meynet \& Maeder (2002), both including stellar rotation (dotted lines) and with no stellar rotation (dashed lines) and with the analytical models of Henry et al. (2000, solid lines) which were computed to fit the N/O ratios observed in extragalactic H II regions (blue compact galaxies). Empty boxes are metal-rich stars listed in Table 2 discussed in the text.

(Fig. 3), the scatter about the fitting line is larger in the [N/O] vs. $[\mathrm{O} / \mathrm{H}]$ plane than in the $[\mathrm{N} / \mathrm{O}]$ vs. $[\mathrm{N} / \mathrm{H}]$ plane. If the scatter were caused purely by observational error, it should be the same for both planes. If, on the other hand, there were intrinsic scatter caused by rotation, the $[\mathrm{O} / \mathrm{H}]$ would not depend on rotation while $[\mathrm{N} / \mathrm{O}]$ would, and this plane shows the full effect of this scatter. Instead both $[\mathrm{N} / \mathrm{H}]$ and $[\mathrm{N} / \mathrm{O}]$ depend on rotation in a similar manner and the resulting scatter is smaller in this plane.

\subsection{Comparison with DLAs}

A large data set of $\mathrm{N}$ abundances in damped Lyman $\alpha$ systems (DLAs) has recently been presented by Centurión et al. (2003). In Fig. 6 we compare our data with that of Centurión et al. (2003, refer to that paper for the original references to all the data). What is plotted is $[\mathrm{N} / \mathrm{Si}]$ since the $\mathrm{Si}$ measurements in DLAs are more reliable than $\mathrm{O}$ measurements. The $[\mathrm{N} / \mathrm{O}]$ ratios of DLAs seem to lie in the same region occupied by Galactic stars with two differences: i) the DLAs extend to lower [N/O] ratios than galactic stars; ii) although there are some DLAs with "high" N/O ratios they never reach the very high values displayed by a few Galactic stars at comparable metallicity. The conclusion is that the $\mathrm{N}$ production history is similar in our Galaxy and DLAs, but different in BCGs.

Among the DLAs there is a group of 5 which have a very low $[\mathrm{N} / \mathrm{Si}](\sim-1.4)$ and Molaro et al. (2003) claim that these represent a second plateau in nitrogen abundance. The proposed interpretation of this is that these galaxies are very young

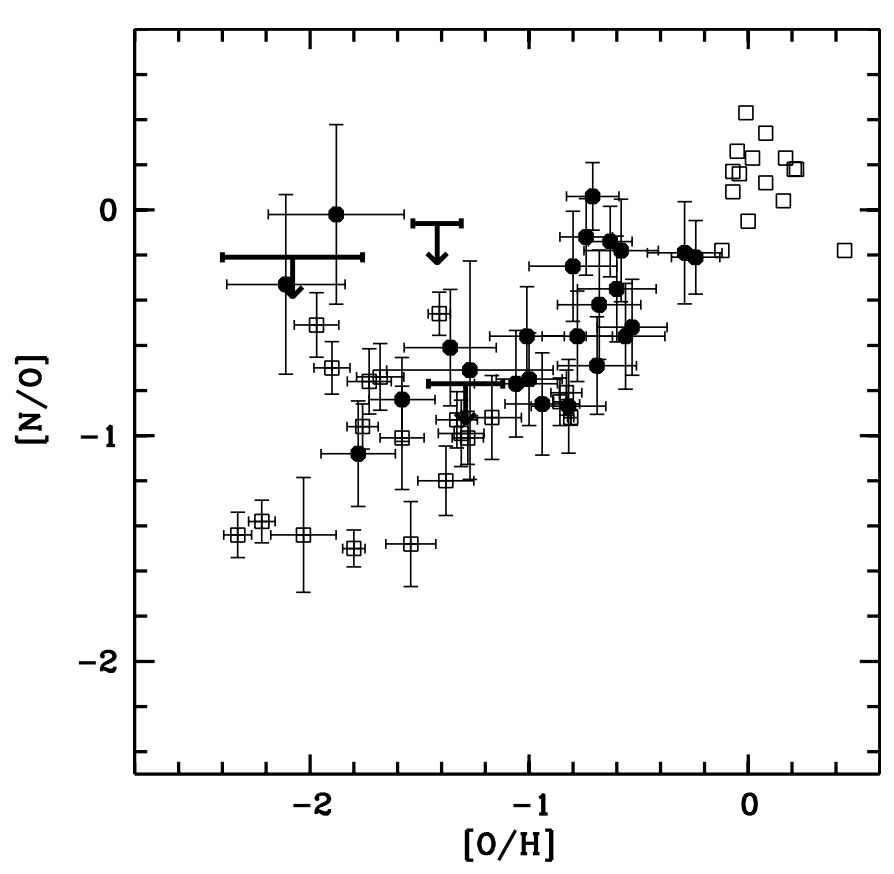

Fig. 6. The $[\mathrm{N} / \mathrm{O}]$ as a function of $[\mathrm{O} / \mathrm{H}]$ for the programme stars (filled circles) and for the damped Lyman $\alpha$ systems (DLAs) from Centurion et al. (2003, crossed squares). We chose to show [N/Si] rather than [N/O] since there are more data points available and the measurements of $\mathrm{Si}$ are more reliable than those of O. However, we should bear in mind that different groups of objects (e.g. DLAs, BCGs and Galactic stars) may have different star formation histories.

and are observed before intermediate mass stars had time to contribute to the $\mathrm{N}$ abundance. If our Galaxy has passed such a phase there should be some stars with such a low $[\mathrm{N} / \alpha]$; moreover, no star with a lower $[\mathrm{N} / \alpha]$ ratio should be observed. The lowest point with $[\mathrm{N} / \mathrm{O}]=-1.08$ corresponds to the star $\mathrm{BD}+23^{\circ} 3130$ and is therefore above the "low $\mathrm{N} / \alpha$ " group of DLAs, although the errors in abundances (of both the stars and the DLAs) are such that the two are consistent at less than $1 \sigma$. It would clearly be of great importance to extend the stellar observations at lower metallicities to see whether the $\mathrm{N} / \mathrm{O}$ ratio persists in its linear decrease or whether it flattens out and reaches values similar to those of the "low $\mathrm{N} / \alpha$ " DLAs.

\subsection{N-rich stars}

Several definitions could be made of what is N-rich or $\mathrm{N}$-normal, and some stars might be classified differently according to the definition chosen. However, two stars in our sample that would surely be ascribed to this group and are G6412 and LP 815-43. Neither of these two well-known metalpoor stars shows any serious chemical anomaly. Both stars have a Li abundance (Bonifacio \& Molaro 1997; Ryan et al. 1999; Bonifacio 2001) that places them on the lithium plateau. This places serious constraints on the process that enriches them in $\mathrm{N}$, since $\mathrm{Li}$ is destroyed at the temperatures at which the $\mathrm{CNO}$ cycle is operating $\mathrm{Li}$. The problem is not new and several other N-rich field stars are known (Bessell \& Norris 1982; Laird 1985), all of which are lithium-normal (Spite \& Spite 1986). Likewise, the globular cluster NGC 6397 is N-rich 
although Li-normal (Bonifacio et al. 2002). In their pioneering survey Carbon et al. (1987) pointed out that the number of $\mathrm{N}$-rich stars is very limited; however, in our example there are at least two clear examples which are similar to the previously five known (HD 25329, HD 74000, HD 97916, HD 160617 and HD 166913). We believe these stars are the exception rather than the rule, although they are perhaps less rare than previously thought.

\subsection{Nitrogen nucleosynthesis and final remarks}

Nitrogen is one of the most abundant elements in the Universe. With $\mathrm{C}$ and $\mathrm{O}$, it shares an important nucleosynthetic role as a key ingredient in hydrogen-burning through the $\mathrm{CNO}$ cycle. However while for carbon and oxygen the dominant production modes are the $\alpha$-chain reactions, which start from the carbonproducing triple- $\alpha$ reaction, for nitrogen the dominant production mode lies in the re-arrangement of nuclei which occurs in the CNO cycle. In CNO-cycled material ${ }^{14} \mathrm{~N}$ is increased at the expense of ${ }^{12} \mathrm{C}$ and ${ }^{16} \mathrm{O}$. However, there is still considerable uncertainty as to where and when this process takes place. Hydrogen-burning operates mainly through the $\mathrm{CNO}$ cycle during the core H-burning (main sequence) phase of stars slightly more massive than the Sun and during shell H-burning phases. The latter include: the first ascent along the red giant branch (RGB), the horizontal branch (HB) and the asymptotic giant branch (AGB). The first question to ask is under which of these conditions the nuclear-processed material is more readily shed into the interstellar medium. Our targets are unevolved dwarfs and therefore the $\mathrm{N}$ and $\mathrm{O}$ abundances discussed in this paper reflect conditions in the ISM at the time of star formation. The second question is where the $\mathrm{C}$ and $\mathrm{O}$ nuclei, used to form $\mathrm{N}$, come from. Were these synthesized by a previous stellar generation or by the star itself?

In standard stellar models the material processed in the core of a massive star does not reach the surface. Therefore, core $\mathrm{H}$-burning should not provide any nitrogen. However, if we add some extra mixing, e.g. because of rotation, a part of the core material may reach the surface (see the discussion in Meynet $\&$ Maeder 2002 and their Table 2).

Concerning shell-H burning, according to the standard models, it is only the AGB phase, through the third dredgeup mechanism in which it is possible to bring nucleosynthesis products to the surface. Also, in this case inclusion of rotation allows surface enrichment even prior to the third dredge-up (Meynet \& Maeder 2002).

The second question we have posed is usually found in the literature as: Is nitrogen primary or secondary? Since nitrogen needs $\mathrm{C}$ and $\mathrm{O}$ to be formed, if nitrogen is formed from the $\mathrm{C}$ and $\mathrm{O}$ already existing at the time the star formed then it is called "secondary". If, instead, the $\mathrm{C}$ and $\mathrm{O}$ are formed in the star itself and then used for $\mathrm{N}$ production then $\mathrm{N}$ is called "primary". According to the standard models, primary production can take place in the AGB phase of stars that undergo hot bottom burning (Marigo 2001); therefore, it takes place in intermediate mass stars. In models which include rotational mixing, production of primary nitrogen can instead take place both in intermediate mass and massive stars. Moreover, in intermediate mass stars the mechanism may be different from that in the standard models: in an $\mathrm{H}$-shell burning star the He-processed material, containing $\mathrm{C}$ and $\mathrm{O}$, may reach the H-burning shell through rotational diffusion, thus giving rise to primary nitrogen.

It thus appears reasonably clear, from the theoretical point of view, that any stellar population will produce some primary, as well as some secondary, nitrogen. We thus expect this element to show a behavior that is neither purely primary nor purely secondary.

Spectral syntheses presented in this article are subject to many uncertainties. Stellar parameters and line lists are questioned first of all. While we did our best to obtain reliable stellar parameters and a well calibrated line list, two more uncertainties could still affect the final results: the effects of granulation/3D and the opacity uncertainties in the near UV. Both effects can be greatly minimized or perhaps even canceled if we measure the (N/O) ratios from the near-UV bands of $\mathrm{NH}$ and $\mathrm{OH}$ and plot them against $[\mathrm{Fe} / \mathrm{H}]$ derived from the Fe II lines (Nissen et al. 2002). However, these effects may not be masked on the graphs which plot $(\mathrm{N} / 0)$ against $(\mathrm{O} / \mathrm{H})$ (or $(\mathrm{N} / \mathrm{H})$ ) since the latter will be affected. While it is still impossible to carry out $\mathrm{NH}$ analysis with 3D models in a large number of metal-poor stars, we can guess what the general effect would be. The 3D effect on $\mathrm{OH}$ increases with decreasing $[\mathrm{Fe} / \mathrm{H}]$ (Asplund \& García Pérez 2001). Thus, the lowest metallicity points in Fig. 5 will be shifted horizontally to the right and the signature of primary Nitrogen found in 1D models (i.e. the flattening at low $(\mathrm{O} / \mathrm{H})$ ) will disappear. Finally, we note that even this "guess" is very uncertain since 3D models still do not resolve the oxygen conflict in metal-poor stars, and more work is needed to achieve consistent abundance analysis.

Acknowledgements. We would like to thank Andre Maeder and Georges Meynet for many important discussions and comments on this paper. The referee Richard Henry is thanked for many valueable suggestions and comments.

\section{References}

Anders, E., \& Grevesse, N. 1989, Geochim. Cosmochim. Acta, 53, 197

Allende Prieto, C., Lambert, D. C., \& Asplund, M. 2001, ApJ, 556, L63

Asplund, M., \& García Pérez, A. 2001, A\&A, 372, 601

Asplund, M., Grevesse, N., Sauval, J., Allende Prieto, C., \& Kiselman, D. 2004, A\&A, 417, 751

Bessell, M. S., \& Norris, J. 1982, ApJ, 263, L29

Bihain, G., Israelian, G., Rebolo, R., Bonifacio, P., \& Molaro, P. 2004, A\&A, accepted

Boesgaard, A. M., King, J. R., Deliyannis, C. P., \& Vogt, S. S. 1999, AJ, 117, 492

Bonifacio, P. 2001, in The link between stars and cosmology, ed. M. Chavez, \& D. Mayya (Dordrecht: Kluwer), 77

Bonifacio, P., Pasquini, L., Spite, F., et al. 2002, A\&A, 390, 91

Bonifacio, P., \& Molaro, P. 1997, MNRAS, 285, 847

Carbon, D. F., Barbuy, B., Kraft, R. P., Friel, E. D., \& Suntzeff, N. B. 1987, PASP, 99, 335 
Centurión, M., Bonifacio, P., Molaro, P., \& Vladilo, G. 1998, ApJ, 509,620

Centurión, M., Molaro, P., Vladilo, G., et al. 2003, A\&A, 403, 55

Ecuvillon, A., Israelian, G., Santos, N., Mayor, M., \& García Lopez, R. J. 2004, A\&A, 418, 703

Edmunds, M. G., \& Pagel, B. E. 1978, MNRAS, 185, 78P

Fulbright, J., \& Johnson, J. 2003, ApJ, 595, 115

García López, R. J., Lambert, D. L., Edvardsson, B., et al. 1998, ApJ, 500, 241

García López, R. J., Severino, G., \& Gomez, M. T. 1995, A\&A, 297, 787

Gonzalez, G., Laws, C., Tyagi, S., \& Reddy, B. E. 2001, AJ, 285, 403

Henry, R. B. C., Edmunds, M. G., \& Köppen, J. 2000, ApJ, 541, 660

Israelian, G., García López, R. J., \& Rebolo, R. 1998, ApJ, 507, 805

Israelian, G., García López, R. J., \& Rebolo, R. 2000, in The Evolution of the Milky Way: stars versus clusters, ed. F. Matteucci, \& F. Giovannelli (Dordrecht: Kluwer), 35

Israelian, G., Rebolo, R., García López, R., et al. 2001, ApJ, 551, 833

Israelian, G., Shchukina, N., Rebolo, R., et al. 2004, A\&A, 419, 1095

Izotov, Y. I., Schaerer, D., \& Charbonnel, C. 2001, ApJ, 549, 878

Kurucz, R. L. 1993, CD-ROMs, ATLAS9 Stellar Atmospheres Programs and $2 \mathrm{~km} \mathrm{~s}^{-1}$ Grid (Cambridge: Smithsonian Astrophys. Obs.)

Kurucz, R. L., Furenlid, I., Brault, J., \& Testerman, L. 1984, Solar Flux Atlas from 296 to $1300 \mathrm{~nm}$, NOAO Atlas No. 1

Laird, J. B. 1985, ApJ, 289, 556

Liang, Y. C., Zhao, G., \& Shi, R. J. 2001, A\&A, 374, 936

Lu, L., Sargent, W., \& Barlow, T. A. 1998, AJ, 115, 55

Maeder, A. 1992, A\&A, 264, 1

Marigo, P. 2001, A\&A, 370, 194
Meynet, G., \& Maeder, A. 2002, A\&A, 390, 561

Molaro, P., Bonifacio, P., Castelli, F., \& Pasquini, L. 1997, A\&A, 319, 593

Molaro, P., Centurión, M., D’Odorico, V., \& Peroux, C. 2003, [arXiv: astro-ph/0307173]

Nissen, P. E., Primas, F., Asplund, M., \& Lambert, D. L. 2002, A\&A, 235, 251

Pagel, B. E. J., \& Edmunds, M. G. 1981, ARA\&A, 19, 77

Pagel, B., \& Kazlauskas, A. 1992, MNRAS, 256, 49P

Pettini, M., Ellison, S. L., Bergeron, J., \& Petitjean, P. 2002, A\&A, 391, 21

Sadakane, K., Ohkubo, M., Takeda, Y., et al. 2002, PASJ, 54, 911

Santos, N. C., Israelian, G., \& Mayor, M. 2000, A\&A, 363, 228

Sargent, W., \& Searle, L. 1970, ApJ, 162, 155

Schulte-Ladbeck, R., Ulrich, H., Greggio, L., Crone, M., \& Drozdovsky, I. 2001, AJ, 121, 3007

Sneden, C. 1974, ApJ, 189, 493

Spite, F., \& Spite, M. 1986, A\&A, 163, 140

Ryan, S. G., Norris, J. E., \& Beers, T. C. 1999, ApJ, 523, 654

Takeda, Y., Sato, B., Kambe, E., et al. 2001, PASJ, 53, 1211

Takeda, Y. 2003, A\&A, 402, 343

Thuan, T. X., Izotov, Y. I., \& Lipovetsky, V. A. 1995, ApJ, 445, 108

Tomkin, J., \& Lambert, L. 1984, ApJ, 279, 220

Tomkin, J., Lemke, M., Lambert, D. L., \& Sneden, C. 1992, AJ, 104, 1568

Yakovina, L. A., \& Pavlenko, Ya. V. 1998, Kinematics and Physics of Celestial Bodies, 14, 195

van den Hoek, L. B., \& Groenewegen, M. A. T. 1997, A\&AS, 123, 305 